\title{
Editorial Comment: Beyond Social Determinants
}

Journal of Public Health Policy (2008) 29, 105.

doi:I0.I057/palgrave.jphp.3200I69

We are particularly pleased to present "The Impact of Corporate Practices on Health: Implications for Health Policy," because we believe it raises important strategic and tactical questions for efforts to improve population health; questions far beyond the scope of the article itself.

We have been encouraged by research that brings nuance to our understanding of the causes of diminished health status of populations - social determinants of health. Income disparities, for example, seem to contribute to poor health of the population. These disparities, and other social determinants of health, tell us a great deal about how and why people are healthy or not, but far less about how to respond.

To alter most social determinants seems to require little short of a revolution. We find the simplicity of a focus on corporate practices encouraging because particular practices, policies, and actions can be prevented, altered, and/or eliminated by direct attention and action. As a matter of strategy for improving health, we think that public health would do well to emulate the authors and look for the best targets, where simple actions can improve population health. 